\title{
GROUPROFILE: Um Modelo de Gerenciamento de Perfis de Grupos de Aprendizes
}

\author{
Adalto Selau Sparremberger ${ }^{1}$, Jorge Luis Victória Barbosa ${ }^{1}$, João Carlos Gluz ${ }^{1}$ \\ ${ }^{1}$ Programa Interdiciplinar de Pós-Graduação em Computação Aplicada - PIPCA \\ Universidade do Vale do Rio dos Sinos - UNISINOS \\ Av. Unisinos, 950 - Cristo Rei - São Leopoldo/RS - CEP: 93022-750 \\ professor@adalto.pro.br, \{jbarbosa,jcgluz\}@unisinos.br
}

\begin{abstract}
The Virtual Learning Environments (VLE) facilitate the activities of Learning and Distance Education (LDE). Although, if initially, VLE creates an easy way to the realization of course in the LDE model, on another hand, can lead to creating gaps among teacher and students. It happens because the VLE courses are made at a distance, without physical contact between the professor and students difficulties the task of to recognize students profile (e.g. characteristics, preferences, problems or feelings). Therefore, this work proposes to address this gap using a model called GrouProfile, which allows management of student groups profiles based on historical contexts, taking account a set of metrics to measure the student's proactivity and reactivity.
\end{abstract}

Resumo. Os Ambientes Virtuais de Aprendizagem (AVA) facilitam as atividades de Aprendizado e Educação a Distância (EaD). Contudo, se por um lado os AVAs facilitam a realização dos cursos EaD, também podem acarretar em um distanciamento entre professor e alunos. Este distanciamento ocorre porque os professores não possuem contato físico com os alunos, acarretando na dificuldade de identificar o perfil (características, gostos, problemas, sentimentos) de cada aluno e da turma. Visando minimizar esta lacuna, este trabalho propõe um modelo denominado GrouProfile. Este modelo permite o gerenciamento de perfis de grupos de aprendizes baseado em históricos de contextos, levando em consideração métricas para mensurar proatividade, reatividade e interesse dos alunos.

\section{Introdução}

Ambientes Virtuais de Aprendizagem (AVA) são ferramentas tecnológicas que foram criadas para apoiar o ensino e a aprendizagem [PENTERICH 2005]. Estas ferramentas são mais conhecidas por sua utilização em cursos oferecidos na modalidade $\mathrm{EaD}$, uma vez que, nesta modalidade os cursos dependem do AVAs para viabilizar a sua execução. Entretanto, estas ferramentas podem e devem ser utilizadas também em cursos presenciais, devido ao fato de propiciarem ao docente armazenar materiais, realizar avaliações, receber trabalhos dos alunos e acompanhar os índices da turma no que se refere a notas, proatividade, reatividade e interesse.

Estes índices são mais facilmente mensurados em cursos ofertados na modalidade presencial, uma vez que, estes cursos se beneficiam da interação diária presencial com 
VI Congresso Brasileiro de Informática na Educação (CBIE 2017)

Anais do XXVIII Simpósio Brasileiro de Informática na Educação (SBIE 2017)

o aluno, propiciando ao docente identificar no comportamento do aluno em sala de aula, situações que caracterizem boa proatividade, reatividade, e alto índice de interesse bem como comportamentos que caracterizem baixos índices destes mesmos critérios.

Desta forma, visando proporcionar ao professor de cursos ofertados na modalidade $\mathrm{EaD}$, a mesma possibilidade de identificar o perfil comportamental dos alunos, os AVA precisam estar constantemente sendo atualizados, revistos, e incrementados. Uma funcionalidade que vem sendo estudada para ser incluída nestes ambientes, é torna-los capazes de permitir ao docente identificar o perfil dos seus alunos, no que diz respeito aos aspectos comuns: como se comportam? Quais são suas angustias, quais suas principais dificuldades, entre outras características formadoras de perfil [Felder and Silverman 1988]. A partir daí, os AVAs devem buscar permitir a identificação de um perfil de consenso, sendo este, o perfil médio construído, ou seja, aquele que reflete uma média do perfil de todos os membros de um grupo pré-estabelecido e.g. uma turma de alunos.

Além de identificar o perfil de consenso é necessário que os AVA armazenem estes valores, criando um histórico de perfils. Isto é importante porque permite ao professor identificar a evolução da turma nos critérios a qual desejar monitorar. A identificação do perfil de consenso e a possibilidade de consultar o histórico dos perfis permite ao professor elaborar materiais, preparar aulas e trabalhos de acordo com as necessidades deste perfil específico. Esta tarefa é de fundamental importância, uma vez que, materiais padronizados e não personalizados para o perfil da turma, faz com que não seja despertado nos alunos o desejo de aprender e ir além dos conteúdos apresentados pelos materiais [de Souza and Gasparin 2012]. Despertar nos alunos a vontade de aprender proativamente é de suma importância uma vez que, uma classe em que os alunos são sempre passivos é uma classe em que nem o experimentador ativo nem o observador reflexivo, podem aprender de forma eficaz [Felder and Silverman 1988].

A perspectiva de melhorar o interesse dos alunos em estudar proativamente, devido a utilização de material didático e aulas personalizadas de acordo com o perfil de consenso da turma, motiva a construir ambientes para o aprendizado de forma virtual que cada vez mais se aproximem de ambientes tradicionais (presencial) sem deixar de prover as facilidades que os AVA promovem (ensino a distância, flexibilidade de horários, entre outros). Neste contexto, buscamos neste trabalho criar um agente de software para o gerenciamento de perfis de grupos de aprendizes, através de históricos de perfil de consenso. Este agente é composto por um monitor de grupos que permite que o professor gerencie e receba notificações de mudanças no perfil no grupo monitorado. Estes perfis são atualizados dinamicamente com base em regras determinadas por um modelo chamado GrouProfile que estabelece a definição de proatividade, reatividade, interesse, grupo de alunos com melhor desempenho e grupo de alunos com desempenho inferior. A partir dos índices individuais e dos perfis de consenso criados, o agente armazena estas informações gerando um histórico da evolução e das mudanças de cada turma.

Para a concepção deste trabalho primeiramente realizou-se uma revisão de literatura, fazendo uso da estratégia Snowballing [Wohlin 2014]. Posteriormente, realizou-se uma survey através de questionários aplicados para professores, buscando responder o que consideram característico de alunos proativos, reativos, de melhor desempenho e de desempenho inferior. E por fim, para validação dos resultados, realizou-se um estudo de caso e aplicou-se novo questionário apenas para os professores das turmas que par- 
VI Congresso Brasileiro de Informática na Educação (CBIE 2017)

Anais do XXVIII Simpósio Brasileiro de Informática na Educação (SBIE 2017)

ticiparam do estudo de caso. Após a realização deste estudo de caso pudemos perceber que o GrouProfile é capaz de estabelecer o perfil de consenso da turma e o perfil individual de cada aluno de forma igual ou bastante similar a visão que o professor de turmas presenciais possui.

\section{Revisão de Literatura}

Na revisão de literatura, foi utilizada a técnica metodológica Snowballing [Wohlin 2014]. Esta estratégia permite a busca de trabalhos baseado nas referências e nas citações de artigos definidos como artigos de controle, ou seja, artigos considerados referência para esta área. $\mathrm{O}$ objetivo desta etapa foi identificar trabalhos realizados por outros pesquisadores, que apresentam contribuições no mesmo ponto focal deste estudo. Neste sentido destacamos os seguintes trabalhos:

- Ferreia et. al (2015) [Ferreira et al. 2015] propõe o trabalho "UbiGroup - Um Modelo de Recomendação Ubíqua de Conteúdo para Grupos de Aprendizes”, este trabalho tem como objetivo desenvolver um método de recomendação ubíqua de objetos de aprendizagem para grupos de aprendizes que possuem o mesmo contexto. Este trabalho relaciona-se com o modelo GrouProfile porque ambos trabalham no contexto de perfil de aprendizes, contudo, o UbiGroup tem foco em recomendação de materiais e não em identificação de proatividade, reatividade e interesse.

- Este trabalho foi posteriormente estendido por Nóbrega et. al (2015) [Nóbrega et al. 2015] no trabalho denominado EduTrail, Um Modelo para Gerenciamento de Perfis de Entidades Através de Inferência em Trilhas. Este modelo permite o acompanhamento ubíquo de grupos de aprendizes realizando um registro histórico dos contextos, a partir de informações provenientes dos temas e tópicos do plano de ensino, dos perfis individuais e de grupos de alunos.

- Barbosa et. al (2008) [Barbosa et al. 2008] apresentaram o modelo entitulado "Local: A Model Geared Towards Ubiquitous Learning". Este trata-se de um modelo para Suporte à Aprendizagem Consciente de Contexto. Este modelo utiliza informações de localização e o gerenciamento de contextos como instrumentos de apoio à educação, auxiliando nos processos de ensino e aprendizagem.

- Levis et. al. (2008) [Levis et al. 2008] apresentam o trabalho: PeLeP - Pervasive Learning Profile que tem por objetivo possibilitar que as aplicações educacionais possam explorar o ensino e aprendizagem ubíquos, baseando-se em um perfil organizado por categorias que contêm informações da vida cotidiana do aprendiz.

- O trabalho "Análise Visual para Monitoramento de Alunos de Cursos a Distância", apresentado por Weiand (2016) [WEIAND 2016], propõe através de algoritmos de visualização de dados, melhorar o acesso do docente a informações referentes aos alunos dos cursos EaD. Contudo, este trabalho não propõe métricas específicas para análise de proatividade, reatividade, interesse e explora a visualização dos dados sem considerar efetuar ranqueamento dos alunos.

- Schmitt (2015) [Schmitt et al. 2010] desenvolveu um plugin para o AVA Moodle chamado Analytics Graphs, este plugin se assemelha ao GrouProfile, uma vez que, também permite a visualização do perfil de alunos em ambiente virtual. Este plugim permite ao professor visualizar o perfil dos alunos de acordo com notas, acesso a arquivos e recursos e envio de tarefas. Contudo, nesta ferramenta estes 
VI Congresso Brasileiro de Informática na Educação (CBIE 2017)

Anais do XXVIII Simpósio Brasileiro de Informática na Educação (SBIE 2017)

quesitos são avaliados de forma individual não sendo cruzados para a medição de proatividade ou reatividade.

\section{Modelo GrouProfile}

A Figura 1 nos traz a representação do modelo GrouProfile que é composto de quatro agentes de software. Estes agentes se comunicam com a base de dados do GrouProfile e com a base de dados do AVA. A seguir, as atribuições de cada agente que compõe o modelo.

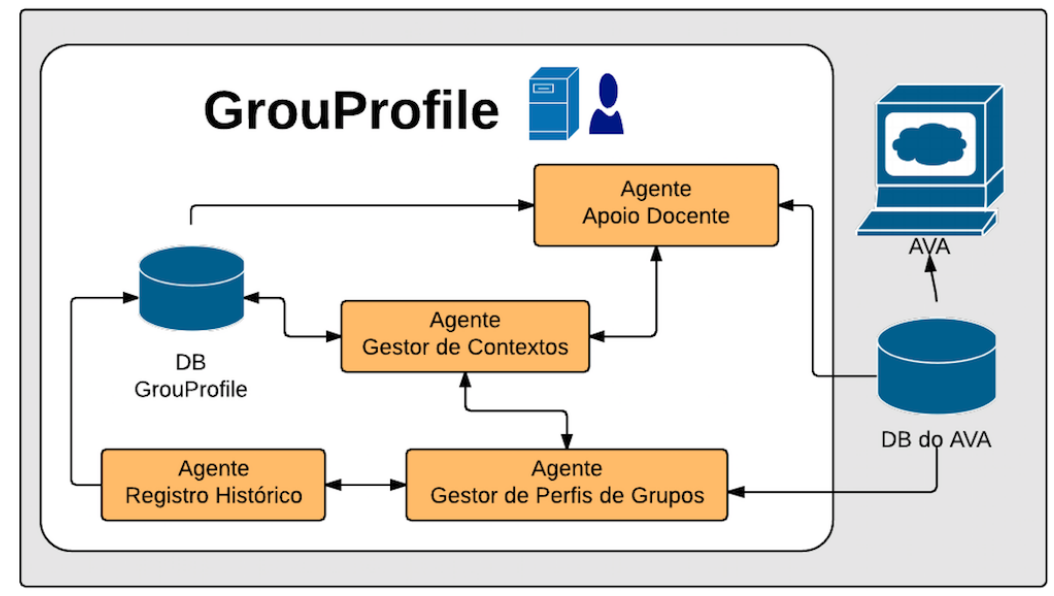

Figura 1. Modelo GrouProfile

\subsection{Agente Apoio Docente (AD)}

O Agente Apoio Docente é inspirado no Agente Apoio Pedagógico, que integra o modelo Ubigroup. Este agente é responsável pela interface com o docente. O AD busca na base de dados do AVA, as credenciais do docente para validação de login. Já na base de dados do GrouProfile, o agente AD busca as informações históricas dos perfis dos grupos.

\subsection{Agente Gestor de Contextos (GC)}

O Agente Gestor de Contextos realiza o monitoramento das atividades dos grupos de aprendizes. É de sua responsabilidade gerenciar os históricos de contextos utilizados pelo GrouProfile.

\subsection{Agente Registro Histórico (RH)}

Inspirado no modelo EduTrail, o Agente Registro Histórico tem por sua responsabilidade o registro dos históricos dos contextos dos grupos de aprendizes, a partir das informações mantidas pelo agente Gestor de Contextos (GC).

\subsection{Agente Gestor de Perfis de Grupos (GPG)}

O agente GPG terá a responsabilidade de se comunicar com os agentes Gestor de Contextos e Registro Histórico, com o propósito de monitorar os grupos de aprendizes, baseandose em análise dos históricos de contextos dos grupos [Dey et al. 2001] [Silva et al. 2009]. O objetivo destas ações, é permitir ao professor que gerencie e receba notificações de movimentações de determinado grupo, através de um monitor de grupos. A cada movimentação e/ou evolução do grupo, seu novo perfil será gerado de forma dinâmica e armazenado, criando assim um histórico de evolução do grupo. 
VI Congresso Brasileiro de Informática na Educação (CBIE 2017)

Anais do XXVIII Simpósio Brasileiro de Informática na Educação (SBIE 2017)

\subsection{Métricas}

GrouProfile é um framework que cria, mantêm e registra histórico do perfil de consenso de grupos de aprendizes. Este perfil de consenso é baseado em proatividade, reatividade e interesse do grupo, sendo que, estes critérios são avaliados conforme métricas pre-definidas pelo modelo GrouProfile.

Para concepção deste modelo, primeiramente buscamos identificar junto a um grupo formado por trinta e seis professores, quais os critérios que estes costumam utilizar para classificação de proatividade, reatividade e interesse entre os grupos de aprendizes dos quais são professores. Para isso realizou-se uma survey, em formato de questionário. Optou-se por utilizar questionário, por este método permitir que o pesquisador receba dados de respondentes que não estão no mesmo espaço físico que o pesquisador, bem como por permitir flexibilidade de horário e privacidade nas respostas.

O questionário foi elaborado levando em consideração dados preliminares coletados em um brainstorm realizado junto aos colegas professores da instituição em que o pesquisador responsável pelo trabalho atua. Este brainstorm foi realizado de forma informal e teve intuito apenas de fornecer informações para estruturação do questionário. Um piloto do questionário foi executado junto a dois professores para validar a estrutura do questionário.As perguntas efetuadas, bem como as alternativas dadas para cada uma delas foram as seguintes:

Questão 01: Quais das seguintes opções caracterizam proatividade de alunos, na sua opinião, tendo como opções: Número de acessos ao ambiente virtual de ensino; Periodicidade de acessos; Acessos ao ambiente virtual efetuados fora do período de provas e trabalhos; Entrega de trabalhos de forma antecipada; Entrega de trabalhos no prazo estabelecido; Número de interações no ambiente virtual (resposta a perguntas, posts efetuados...); Perfil completo no ambiente virtual (foto perfil, nome completo, telefone, perfil rede social ); outros. As respostas obtidas foram as seguintes:

Número de interações no ambiente virtual, $75 \%$.

Acessos ao ambiente virtual efetuados fora do período de provas e trabalhos, 55,6\%;

Entrega de trabalhos no prazo estabelecido,38,9\%;

Periodicidade de acessos, 38,9\%;

Entrega de trabalhos de forma antecipada, 33,3\%;

Número de acessos ao ambiente virtual de ensino, 19,4\%.

OBS: Por tratar de uma questão de múltipla-escolha os percentuais não são somatórios, eles representam o número de respostas dadas entre os respondentes para cada uma das alternativas.

Questão 2: Quais das seguintes opções caracterizam reatividade de alunos em ambientes virtuais, na sua opinião, tendo como opções de resposta: Número de comentários em postagens de colegas (Fórum de Discussões); Número de comentários em dúvidas postadas por colegas no fórum de dúvidas; Percentual de respostas de atividades; Respostas de E-mails; outros. As respostas obtidas foram as seguintes:

Número de comentários em postagens de colegas (Fórum de Discussões), 74,3\%; 
VI Congresso Brasileiro de Informática na Educação (CBIE 2017)

Anais do XXVIII Simpósio Brasileiro de Informática na Educação (SBIE 2017)

Número de comentários em dúvidas postadas por colegas no fórum de dúvidas, 54,3\%;

Percentual de respostas de atividades, $40 \%$;

Respostas de E-mails, 28\%.

Questão 3: Quais das seguintes opções caracterizam interesse de alunos em ambientes virtuais, na sua opinião, tendo como alternativas de respostas:Entrega de todos trabalhos e no prazo; Número de iterações no ambiente virtual maior que a média de todos acessos da turma; Número de dúvidas enviadas ao professor; Número de acessos a materiais extra; outros. As respostas obtidas foram as seguintes:

Entrega de todos trabalhos e no prazo, 44,4\%;

Número de iterações no ambiente virtual maior que a média de todos acessos da turma, $61,1 \%$;

Número de dúvidas enviadas ao professor, 30,6\%;

Número de acessos a materiais extra, $55,6 \%$.

Questão 4: Como você classificaria o grupo de alunos com maior desempenho, tendo como alternativas: Pelo índice de proatividade, reatividade, interesse e pelas notas; Apenas pelas notas; Apenas pela proatividade e interesse; Tendo proatividade e interesse peso superior a nota; Tendo nota pelo superior a proatividade e interesse. As respostas obtidas foram as seguintes:

Pelo índice de proatividade, reatividade, interesse e pelas notas - 80\%;

Apenas pelas notas, $2,8 \%$;

Apenas pela proatividade e interesse $8,3 \%$;

Proatividade e interesse peso superior a nota, $33,3 \%$

Nota peso superior a proatividade e interesse. $22,2 \%$.

\subsection{Definição das Métricas que Compõe o Modelo}

Baseado no resultado do Survey que foi descrito na seção anterior, elaboramos as métricas que são utilizadas para medição de proatividade, reatividade e interesse. A seguir realizamos a análise de cada um destes critérios.

\subsubsection{Definição de Proatividade}

Uma interação proativa segundo Amaral (2011) [Amaral et al. 2011], ocorre quando o aluno vai além do proposto pelo docente, selecionado ou respondendo as estruturas existentes, construindo assim situações únicas e elaborações que forçam os limites do sistema.

Para a definição das métricas de proatividade nos baseamos na questão de número 1(um) do questionário, e visto que, a alternativa "Número de iterações no ambiente virtual" foi a melhor pontuada, elaborou-se a seguinte métrica:

- Número de iterações no ambiente virtual maior ou igual a média da turma: 3 pontos;

- Periodicidade de acessos ao ambiente virtual - 3 pontos

Alunos que efetuam acesso apenas me período de até 24 horas antes de entregas de tarefas contabilizam 1 ponto. 
VI Congresso Brasileiro de Informática na Educação (CBIE 2017)

Anais do XXVIII Simpósio Brasileiro de Informática na Educação (SBIE 2017)

Alunos que acessam o ambiente continuamente, 2 pontos.

Alunos que acessam continuamente e no período de até 24 horas após o professor ter lançado uma tarefa, 3 pontos.

- Entrega de trabalhos - 3 pontos

Alunos que efetuam entregas das tarefas no período das primeiras 24 horas prazo, 3 pontos.

Alunos que efetuam entregas na primeira metade do prazo, 2 pontos.

Alunos que efetuam entregas na segunda metade do prazo, 1 ponto.

- Número de acessos - 0,5 Pontos

Alunos com número de acesso superior a média, 0,5 ponto

- Cadastro Completo - 0,5 Ponto

Alunos com cadastro completo, 0,5 ponto

\subsubsection{Definição de Reatividade}

Conforme Amaral (2011) [Amaral et al. 2011], uma interação reativa é a resposta ao estímulo do sistema sobre o usuário, ou resposta para a questão colocada. O material disponibilizado pelo professor, guia o aluno através de um fluxo pré-determinado.

Para a definição das métricas de reatividade, nos baseamos na resposta na segunda questão do questionário, devido ao fato de Número de comentários em postagens de colegas e Percentual de respostas de atividades serem os itens mais pontuados definiu-se a seguinte métrica.

- Número de acessos ao curso:

Pontuação proporcional a quantidade de acessos menor que a média de todos acessos do grupo, até 1 ponto.

- Número de comentários em postagens de colegas e Número de comentários em dúvidas postadas por colegas - 3 pontos

- Entrega de trabalhos e atividades - 2 pontos

Cálculo proporcional ao total de trabalhos e atividades entregues no prazo.

- Postagem em chat 2 pontos:

Cálculo de percentual de postagens até a média do total de postagens em chat do grupo.

- Envio de e-mail - 2 ponto

Acima da média da turma - 2 pontos

\subsubsection{Definição de Interesse}

Para a definição das métricas de interesse, nos baseamos na resposta da terceira pergunta do questionário. Neste caso as alternativas que foram avaliadas como característica de interesse pelos profissionais que responderam o questionário foram: "Número de iterações no ambiente virtual maior que a média de todos acessos da turma e "Número de acessos a materiais extra. Desta forma a métrica estabelecida valoriza mais estes critérios, sendo ela a seguinte:

- Número de iterações no ambiente virtual maior que a média de todos acessos da turma - 3 pontos 
VI Congresso Brasileiro de Informática na Educação (CBIE 2017)

Anais do XXVIII Simpósio Brasileiro de Informática na Educação (SBIE 2017)

- Número de dúvidas enviadas ao professor maior do que a média da turma - 1

- Número de acessos a materiais extra maior do que a média da turma - 3 pontos

- Entrega de trabalhos e atividades - 3 pontos

Alunos que efetuam entregas na primeira metade do prazo, 3 pontos

Alunos que efetuam entregas na segunda metade do prazo, 2 pontos

Alunos que atrasam entregas de trabalhos, não pontuam

\subsubsection{Definição de alunos com maior desempenho e alunos com menor desempenho}

A métrica que mensura alunos com maior e menor desempenho foi criada baseada na pergunta de número quatro do questionário, para qual $80 \%$ dos respondentes consideraram "Pelo índice de proatividade, reatividade, interesse e pelas notas". Desta forma, estes quatro critérios foram considerados para formulação da métrica de ranqueamento dos alunos de acordo com desempenho, sendo ela a seguinte:

- PRI: (Proatividade * 0.4) + (Reatividade * 0.3) + (Interesse * 0.3)

- Índice Geral: (Notas * 0.6) + (PRI*0.4)

\section{Implementação do Protótipo de Prova}

Como parte da avaliação do modelo proposto pelo GrouProfile, foi desenvolvido um protótipo, utilizando-se da linguagem de programação web $\mathrm{PHP}^{1}$. O banco de dados utilizado para armazenagem do histórico do contexto de cada aprendiz foi o MySQL. Para construção das telas do protótipo e aperfeiçoamento do layout, foi utilizado BootStrap ${ }^{2}$, que é um framework para desenvolvimento de $\mathrm{HTML}^{3}, \mathrm{CSS}^{4}$ e JavaScript.

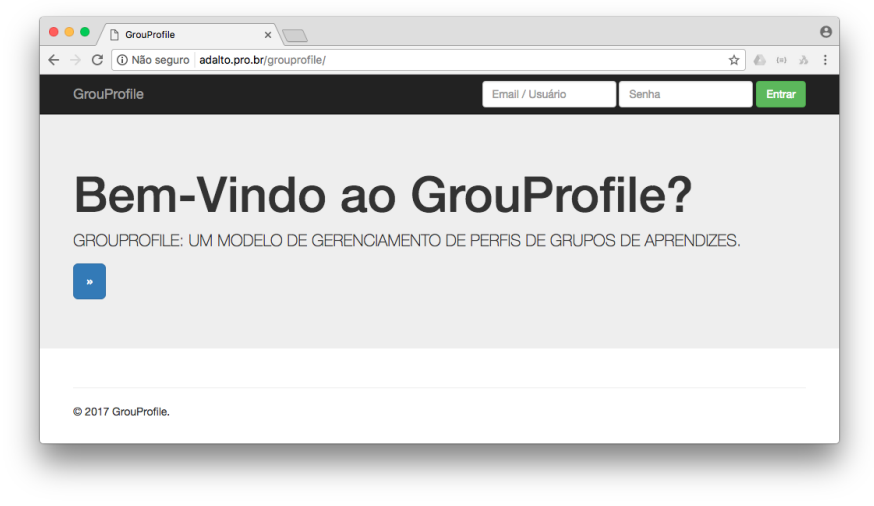

Figura 2. Tela inicial do protótipo GrouProfile

A Figura 2 apresenta a tela inicial do protótipo, que está disponível no endereço http://adalto.pro.br/grouprofile/. O AVA utilizado no experimento foi o Moodle e está disponível em http://adalto.pro.br/moodle/. O funcionamento do protótipo se dá pela análise

\footnotetext{
${ }^{1}$ PHP: Hypertext Preprocessor - Linguagem de programação interpretada, utilizada para desenvolvimento para a web.

${ }^{2}$ http://getbootstrap.com.br/

${ }^{3}$ HTML (HyperText Markup Language) - linguagem de marcação utilizada na construção de páginas na Web.

${ }^{4}$ CSS (Cascading Style Sheets) Estrutura de formatação de estilo (cores, fontes, espaçamento etc) de um documento web.
} 
VI Congresso Brasileiro de Informática na Educação (CBIE 2017)

Anais do XXVIII Simpósio Brasileiro de Informática na Educação (SBIE 2017)

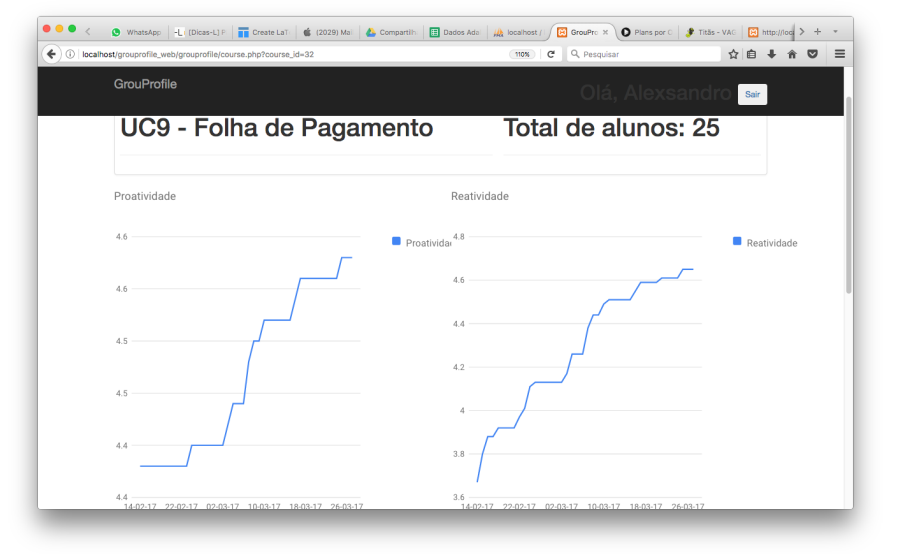

Figura 3. Tela Curso do protótipo GrouProfile

dos logs gerados e armazenados no banco de dados do AVA. O resultado desta análise é armazenada do banco de dados do GrouProfile pelo Agente Registro Histórico. Na figura 3 está representada a tela que contém os gráficos de proatividade, reatividade e interesse do grupo de aprendizes.

\section{Validação do Projeto}

Uma vez concluído o trabalho de desenvolvimento do protótipo de prova, foi realizado um estudo de caso com turmas do curso técnico em administração na modalidade presencial. Optou-se por realizar a validação das métricas propostas e do software com alunos da modalidade presencial, embora o modelo tenha por objetivo principal ser utilizado para curso EaD, uma vez que, objetiva-se que o professor das modalidades EaD possa ter a mesma percepção da proatividade, reatividade e interesse dos alunos do que o professor que trabalha na modalidade presencial tendo contato diário com os alunos. Este estudo foi efetuado entre os meses de dezembro de 2016 e fevereiro de 2017 e executado com grupos de aprendizes de aproximadamente 20 pessoas, totalizando entre os dois grupos, quarenta aprendizes envolvidos.

Ao final do curso o professor respondeu a um questionário que versa sobre a sua percepção em relação ao ranking dos cinco alunos mais proativos, cinco alunos mais reativos, cinco alunos mais interessados, cinco alunos com melhor desempenho e cinco alunos com pior desempenho. As respostas do professor foram confrontadas com as respostadas dadas pelo monitor de perfil de consenso, e desta forma pudemos validar se as métricas definidas permitem ao monitor de consensos uma resposta similar ou idêntica a percepção do professor de cursos presenciais.

\section{Conclusão}

Este trabalho apresentou o desenvolvimento de um modelo e um protótipo, com o propósito de criação de agentes de software para gerenciamento de perfis de grupos de aprendizes em ambientes virtuais de aprendizagem. O protótipo foi utilizado em cursos presenciais onde foi utilizado o AVA Moodle, como ferramenta de apoio ao docente e ao corpo discente. Com o propósito de confrontar as percepções do docente com as informações de perfil do grupo, gerado pelo protótipo. Estas informações do perfil do 
VI Congresso Brasileiro de Informática na Educação (CBIE 2017)

Anais do XXVIII Simpósio Brasileiro de Informática na Educação (SBIE 2017)

grupo são referentes a proatividade, reatividade e interesse. Com base no confronto efetuado, identificamos que o protótipo pode simular a percepção do docente.

\section{Referências}

Amaral, É., Ávila, B., Zednik, H., and Tarouco, L. (2011). Laboratório virtual de aprendizagem: uma proposta taxonômica. RENOTE, 9(2).

Barbosa, J., Hahn, R., Rabello, S., and Barbosa, D. (2008). Local: A model geared towards ubiquitous learning. pages 432-436.

de Souza, M. M. P. and Gasparin, J. L. (2012). A relação professor-aluno e o processo de interação na educação à distância. In Proceedings of XVI Encontro Nacional de Didática e Práticas de Ensino - UNICAMP, pages 13-22, Campinas, São Paulo, Brasil.

Dey, A. K., Abowd, G. D., and Salber, D. (2001). A conceptual framework and a toolkit for supporting the rapid prototyping of context-aware applications. Hum.-Comput. Interact., 16(2):97-166.

Felder, R. M. and Silverman, L. K. (1988). Learning and teaching styles in engineering education. Engineering education, 78(7):674-681.

Ferreira, L. G. A., Barbosa, J. L. V., Gluz, J. C., and Vicari, R. (2015). Ubigroup: Um modelo de recomendação ubíqua de conteúdo para grupos dinâmicos de aprendizes. Revista Brasileira de Informática na Educação, 23(03):40.

Levis, D., Barbosa, J. L. V., Pinto, S. C. S., and Barbosa, D. (2008). Aperfeicoamento automatico do perfil do aprendiz em ambientes de educacao ubiqua. Revista Brasileira de Informática na Educação, 16(1):29-41.

Nóbrega, A. F. d., Barbosa, J. L. V., and Barbosa, D. N. F. (2015). Um modelo para gerenciamento de perfis de entidades através de inferência em trilhas.

PENTERICH, E. (2005). Ambientes virtuais de aprendizagem. Sala de Aula e Tecnologias.

Schmitt, M. A. R., Tarouco, L. M. R., Okuyama, F. Y., Zielinski, F. D. C., and Franco, M. H. I. (2010). Uma ferramenta de learning analytics para o moodle.

Silva, J., Rosa, J. a., Barbosa, J., Barbosa, D. N. F., and Palazzo, L. A. M. (2009). Content distribution in trial-aware environments. In Proceedings of the XV Brazilian Symposium on Multimedia and the Web, WebMedia '09, pages 15:1-15:8, New York, NY, USA. ACM.

WEIAND, A. (2016). ANÁLISE VISUAL PARA MONITORAMENTO DE ALUNOS DE CURSOS À DISTÂNCIA. PhD thesis, Pontifícia Universidade Católica do Rio Grande do Sul.

Wohlin, C. (2014). Guidelines for snowballing in systematic literature studies and a replication in software engineering. In Proceedings of the 18th International Conference on Evaluation and Assessment in Software Engineering, EASE' 14, pages 38:1-38:10, New York, NY, USA. ACM. 